\title{
Characteristic Research of Single-Phase Grounding Fault in Small Current Grounding System based-on NHHT
}

\author{
Yingwei Xiao \\ School of Electrical Engineering and Automation, Hefei University of Technology, Hefei, 230009, China \\ E-mail: xiaoyingwei8@126.com, xiaobenxian@126.com
}

\begin{abstract}
Transient analys is is carried out for the singlephase grounding fault in small current grounding system, the transient grounding current expression is derived, and the influence factors are analyzed. Introduces a method for non-stationary and non-linear signal analysis method -Hilbert Huang transform (HHT) to analyze the single phase grounding fault in small current grounding system, HHT can be better used to extract the abundant transient time frequency information from the non-stationary and nonlinear fault current signals. The empirical mode decomposition (EMD) process and the normalized Hilbert Huang transform (NHHT) algorithm are presented, NHHT is used to analyze and verify an example of the nonlinear and non-stationary amplitude modulation signals. Build a small current grounding system in the EMTP_ATP environment, by selecting the appropriate time window to extract the transient signals, NHHT is used to analyze the transient current signals, and the Hilbert amplitude spectrum and the Hilbert marginal spectrum of the zero sequence transient current signals are obtained. Finally, the influences of the fault phase and the grounding resistance on the time-frequency characteristics of the signals are analyzed.
\end{abstract}

Index Terms-Transient component of zero-sequence current, fault phase, grounding resistance, NHHT, intrinsic mode function (IMF), EMD, Hilbert marginal spectrum.

\section{INTRODUCTION}

At present, the most widely used signal processing method is Fourier transform, but since Fourie r transform can only be applied to linear superposition of FM signal, it can not do time frequency analysis, in order to solve this problem, wavelet transform came into being.

The wavelet transform proposed by Morlet et al., through the expansion and translation of the signal to carry out multi-scale decomposition, can effectively obtain all kinds of time-frequency information from the signal, it has good localization property in time domain and frequency domain, and has the characteristic of multi-resolution analysis.

However, wavelet analysis is not adaptive, once the wavelet base and the decomposition scale are chosen, it is used to analyze the data with multiple frequency components, the results can only reflect the signal characteristics of a fixed time-frequency resolution, and wavelet analysis is not suitable for non-stationary data.

A new signal processing method -- Hilbert Huang transform[1], is proposed for the non-stationary signal by Norden E. Huang et al. In 1998, this method is considered to be a major breakthrough in the linear and steady-state analysis of the Fourier transform.

Norden E. Huang at first proposed the HHT to analyze ocean current signal and wave signal data, and proved its advantages in the analys is of non-stationary and nonlinear signal processing field. Since then, he has used HHT to analyze seismic wave data, which greatly inspired many earthquake researchers and applied it to the field of earthquake research[2-3].

The application of HHT is very extensive, such as Analysis of earth's inner force wave in the field of geophysics[4], structural identification and modal analysis in structural analysis[5], the analysis of solar radiation changes in the field of astrophysics[6], the estimation of Teager energy by using the Hilbert-Huang transform[7], and in [8], HHT is used as an adaptive method to study their multiple scale dynamics in Marine environmental time series, and so on. Its good timefrequency characteristics are demonstrated.

In the biomedical field, empirical mode decompos ition method is used to analyze the electrogastrogram in [9], empirical mode decomposition is used to analyze heart rate variability \& blood pressure in [10], the HHT is used to extract new heath indicators from stationary/ nonstationary vibration signals in [11], Hilbert-Huang transform (HHT) is used to improve the spectrum estimates of heart rate variability (HRV) and extract the features of HRV signals in [12].

In the field of fault processing, the applicability of Hilbert-Huang transform (HHT) for internal leakage fault detection in valve-controlled hydraulic actuators is investigated in [13], the HHT is proposed to detect the very early stage fault in interturn insulation by only analyzing the stator current in the PMSW G in [14], fault detection is realized by means of HHT of the stator current in a PMSM with demagnetization in [15]. Also, Wavelet and Artificial Neural Networks are used to Fault diagnosis [16-17].

In the field of energy, Hilbert spectral analysis (HSA) 
is used to characterize the time evolution of nonstationary power system oscillations following large perturbations in [18], also HHT is used to analyze the nonstationary power-quality waveforms in [19].

The core part of HHT algorithm is empirical mode decomposition (EMD). In essence, EMD is to gradually decompose the oscillation mode or trend of different scale in signal, and produces a series of time series with different characteristic scales, and each time is an IMF component of intrinsic mode function. With the help of Hilbert transform (HT), the time spectrum of signal can be further obtained, which can accurately reflect the timefrequency characteristics of the signal.

In the system of $3 \sim 66 \mathrm{kV}$ power system in which the neutral point is not connected to ground or connected to ground through the arc suppression coil, when the ground fault occurs in a certain phase, because the short circuit can not be formed, the fault current is often much smaller than the load current, this phenomenon is called "small current grounding system". The practice shows that the occurrence of single-phase grounding fault in the power grid is the highest, which is about $85 \%$ of the total failure of the system.

Because the fault current is small in the small current grounding power system, and the detection is difficult, and has not been well solved, which has become one of the research hotspots in this field. In this paper, HHT is used to analyze the fault current of single-phase ground fault in small current grounding system.

The main research work of this paper is as follows:

(1) The transient analysis of the grounding current is carried out and the influence factors are analyzed, the grounding current is mainly transient capacitance current and transient inductance current, but because of the difference between the two frequencies, it can not be offset each other.

(2) The empirical mode decomposition (EMD) process and the normalized Hilbert transform (NHT) algorith $\mathrm{m}$ are presented. The NHHT is used to analyze and verify the nonlinear and non-stationary amplitude modulation signals.

(3) Under the EMTP_ATP environment, a small current grounding system is built, and the parameters of the system are calcu lated, and the single-phase grounding fault is simulated.

(4) Extracts transient signal, use HHT to analyze transient current signal, and obtain time spectrum and Hilbert marginal spectrum of Zero sequence transient current signal of fault feeder, and the influence of the fault phase and the grounding resistance on the timefrequency characteristics of the signal is analyzed.

\section{TRAnSiENT ANALysis OF Single PHASE GROUNDING FAULT IN SMALL CURRENT GROUNDING SYSTEM}

When single phase grounding fault occurs in the neutral point via the arc suppression coil grounding grid, transient capacitance current and transient inductance current constitute the transient grounding current flowing through the fault point. But because of the difference between the two frequencies, the transient process can not compensate for each other. The equivalent circuit of system transient current for the neutral point via the arc suppression coil grounding grid is shown in Fig.1.

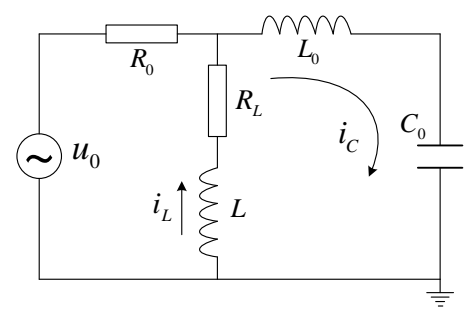

Fig.1. Transient equivalent circuit of single phase grounding fault

In Fig. 1, $C_{0}$ is the equivalent capacitance of the grid to the ground, $L_{0}$ is the equivalent inductance of the circuit, power supply, and the transformer in the zero sequence circuit. $R_{0}$ is the equivalent resistance of the zero sequence circuit, including the grounding resistance of the fault point and the arc path resistance. $R_{L}$ and $L$ are respectively the active loss resistance and inductance of the arc suppression coil, $u_{0}$ is zero sequence power supply voltage.

\section{A. Transient capacitance current}

Since the self vibration frequency of the capacitance current is generally higher, and the inductance of the arc suppression coil is $L \gg L_{0}$, so the influence of $R_{L}$ and $L$ in Fig.1 is neglected in analyzing its transient characteristics. By using the equivalent circuit of $L_{0} \&$ $C_{0} \& R_{0}$ and the system zero sequence voltage source $u_{0}=U_{\Phi \mathrm{m}} \sin (\omega t+\varphi)$, transient capacitance current $i_{C}$ can be obtained. From Figure 1, the differential equation can be got:

$$
R_{0} i_{C}+L_{0} \frac{\mathrm{d} i_{C}}{\mathrm{~d} t}+\frac{1}{C_{0}} \int_{0}^{t} i_{C} \mathrm{~d} \tau=u_{0}
$$

When the grounding resistance is very small, at $R_{0}<2 \sqrt{L_{0} / C_{0}}$, the system is in a state of under damping condition, the transient capacitance current has a periodic oscillation and attenuation characteristics. Transient self vibration component $i_{\text {C.os }}$ and steady state power frequency component $i_{\text {C.st }}$ constitute transient capacitance current $i_{C}$, by using the relationship between $i_{\text {C.os }}+i_{\text {C.st }}=0$ and $I_{\mathrm{Cm}}=U_{\Phi \mathrm{m}} \omega C_{0}$ at initial conditions $t=0$, the following equation can be got by Laplasse transformation:

$$
\begin{aligned}
& i_{\mathrm{C}}=i_{\mathrm{C} . \mathrm{os}}+i_{\mathrm{C} . \mathrm{st}} \\
& =I_{\mathrm{Cm}}\left[\left(\frac{\omega_{f}}{\omega} \sin \varphi \sin \omega t-\cos \varphi \cos \omega_{f} t\right) e^{-\delta t}+\cos (\omega t+\varphi)\right]
\end{aligned}
$$


In the formula (2): $U_{\Phi \mathrm{m}}$ is the amplitude of the phase voltage, $I_{\mathrm{Cm}}$ is the amplitude of the capacitor current, $\omega_{f}$ is the transient self-vibration angular frequency, $\delta=1 / \tau_{\mathrm{C}}=R_{0} / L_{0} / 2$ is the attenuation coefficient of free oscillating component, where the $\tau_{\mathrm{C}}$ is the time constant of the loop. When $\tau_{\mathrm{C}}$ is larger, the self - vibration attenuation is slower, whereas, the attenuation is faster.

By analy zing formula (2), if the single phase grounding fault occurs in $\varphi=\pi / 2$, and at $t=T_{f} / 4\left(T_{f}=2 \pi / \omega_{f}\right.$ is self vibration period), the amplitude of the self vibration part of the capacitive current is the maximum $i_{\text {C.os max }}$ :

$$
i_{\text {C.os } \max }=I_{\mathrm{Cm}} \frac{\omega_{f}}{\omega} e^{-\frac{T_{f}}{4 T_{\mathrm{C}}}}
$$

If the single phase grounding fault occurs in $\varphi=0$, and at $t=T_{f} / 2$, the minimum value of $i_{\text {C.os min }}$ for the self vibration current is:

$$
i_{\text {C.os } \min }=I_{\mathrm{Cm}} \frac{\omega_{f}}{\omega} e^{-\frac{T_{f}}{2 T_{\mathrm{C}}}}
$$

Self-vibration frequency $\omega_{f}$ of self-vibration current is:

$$
\omega_{f}=\sqrt{\omega_{0}^{2}-\delta^{2}}=\sqrt{\frac{1}{L_{0} C_{0}}-\left(\frac{R_{0}}{2 L_{0}}\right)^{2}}
$$

In formula (2), Self-vibration frequency of the loop is $\omega_{0}=\sqrt{1 / L_{0} / C_{0}}$. When the grounding resistance increases to $R_{0} \geq 2 \sqrt{L_{0} / C_{0}}$, the system is in a state of over damping state, and the loop current has a non periodic oscillation attenuation characteristic and tends to be stable.

According to the above analysis, the change of the fault resistance has a great influence on the transient characteristics of the grounding system of the neutral point via arc suppression coil.

\section{B. Transient inductance current}

Differential equation of the core flux can be derived from Fig. 1 [1]:

$$
u_{0}=R_{L} i_{L}+W \frac{\mathrm{d} \psi_{L}}{\mathrm{~d} t}
$$

In the formula (6), $W$ is the turns of the arc suppression coil, and $\psi_{L}$ is the core flux of the arc suppression coil.

Before the grounding fault, no current flows through the arc suppression coil, that is, $\psi_{L}$ is zero. Take $i_{L}=W \cdot \psi_{L} / L$ into formula (6), $\psi_{L}$ expression can be obtained as follow:

$$
\psi_{L}=\Psi_{s t} \frac{\omega L}{Z}\left[\cos (\varphi+\xi) e^{\sqrt{\frac{t}{\tau_{L}}}}-\cos (\omega t+\varphi+\xi)\right]
$$

In the formula (6): $\Psi_{s t}=U_{\Phi \mathrm{m}} /(\omega W)$ is the steady state flux, $\xi=\arctan \left[R_{L} /(\omega W)\right]$ is the phase angle of compensating current, $Z=\sqrt{R_{L}^{2}+(\omega L)^{2}}$ is the arc suppression coil impedance, $\tau_{L}$ is the time constant of the inductance loop. Due to $R_{L} \ll \omega L$, take $Z \approx \omega L$, $\xi=0$. In consideration of $\psi_{L}=\psi_{o s}+\Psi_{s t}, i_{L}=i_{L \cdot d c}+i_{L \cdot s t}$ and $I_{L m}=U_{\Phi m} /(\omega L)$, the expression of the transient inductance current $i_{L}$ can be obtained as follow:

$$
i_{L}=I_{L m}\left[\cos \varphi e^{\frac{-t}{\tau_{L}}}-\cos (\omega t+\varphi)\right]
$$

By the formula (8), it is known that the transient DC component and the steady-state AC component constitute the transient inductance current $i_{L}$, and the initial amplitude of the transient DC decay process is related to the phase $\varphi$, the attenuation coefficient is determined by loop impedance. Transient DC component in the $\varphi=0$ is the largest, and in the $\varphi=\pi / 2$ is the smallest.

\section{Transient grounding current}

System transient grounding current is composed of transient capacitance current $i_{C}$ and transient inductance current $i_{L}$, although the amplitude difference between $i_{C}$ and $i_{L}$ is not large, they are in different frequency bands and can not compensate for each other.

The expression of the transient grounding current $i_{d}$ can be derived from the formula (2) and (8):

$$
\begin{aligned}
& i_{d}=i_{C}+i_{L}=\left(I_{C m}-I_{L m}\right) \cos (\omega t+\varphi) \\
& +I_{C m}\left(\frac{\omega_{f}}{\omega} \sin \varphi \sin \omega t-\cos \varphi \cos \omega_{f} t\right) e^{-\frac{t}{\tau_{C}}}+I_{L m} \cos \varphi e^{-\frac{t}{\tau_{L}}}
\end{aligned}
$$

In the formula (9), the first part of equality right is the steady-state component of the grounding current, the amplitude is the difference between the amplitude of the steady state capacitance current and the steady state inductance current, and is closely related to the fault phase $\varphi$, frequency is power frequency. The other part is the transient component of the grounding current, which is composed of two parts, transient free oscillation component of capacitance current and DC decaying component of inductance current.

From the above analysis, after the occurrence of a single phase grounding fault, transient grounding current flowing through the fault point contains transient capacitive current component of damped oscillation and the decay transient inductance current component. Whether the system is operated in the neutral point via 
the arc suppression coil grounding mode or not grounding mode, the transient capacitance current dominates the amplitude and frequency of the transient grounding current, and its amplitude is also related to the fault phase. The initial value of DC component in the transient inductance current is related to the initial phase and core saturation.

\section{HILBERT HUANG TRANSFORM (HHT)}

\section{A. Instantaneous frequency and intrinsic mode function (IMF)}

Instantaneous frequency is one of the most important concepts in time frequency analysis. The definition of instantaneous frequency is needed to satisfy a certain condition, and not all the instantaneous frequency of the signal has a physical meaning, only when the signal is approximated by a single component signal, each moment corresponds to a single frequency component, the instantaneous frequency has the actual physical meaning. In order to obtain the instantaneous frequency of physical meaning, Norden.E.Huang et al. proposed the concept of intrinsic mode function (IMF).

Using Hilbert transform to analyze the intrinsic mode function (IMF) can get good time frequency characteristics, but most of the signal is not the intrinsic mode function (IMF), and is the combination of the many of intrinsic mode function (IMF). Therefore, the correct time frequency characteristics can not be obtained directly through the Hilbert transform, so that we can not accurately analyze the signal directly. In order to use the Hilbert transform to analyze the nonlinear and nonstationary signals correctly, the signal is decomposed to IMF at first, then the empirical mode decomposition (EMD) is developed.

\section{B. The principle of empirical mode decomposition (EMD)}

Empirical mode decomposition (EMD) uses selection after layer by layer, and get a series of intrinsic mode functions (IMF), and the dominant frequency of the intrinsic mode function (IMF) is lower and lower, the dominant frequency of IMF obtained firstly is the highest, and the IMF obtained lastly is the lowest, therefore, it is essentially a filter bank.

If a signal with random noise is decomposed by the empirical mode decomposition (EMD), the high frequency intrinsic mode function (IMF) component is usually a high frequency noise of the signal, and the residual signal component is usually the mean or trend of the original signal. Each intrinsic mode function (IMF) component obtained from decomposition has obvious physical meaning and contains a certain range of characteristic scales.

The intrinsic mode function (IMF) must meet the signal of the following two conditions [1]:

(1) The zero pole points in the signal are equal or have difference at most 1 .

(2) Any point on the signal, the mean value of the envelope which are determined by local maximum point and local minimum point is zero, that is, the signal is locally symmetric about the time axis. IMF is essentially a narrow band signal.

Because any given signal can not satisfy the IMF condition, the complex signal is decomposed into a series of IMF by EMD[20]. For a given signal $X(t)$, the EMD process is as follows [21]:

Step1. finds out all the local maxima and minima of $X(t)$, by using the three spline interpolation, the local extreme value is connected to the upper and lower two envelope of $e_{\min }(t)$ and $e_{\max }(t) \cdot X(t)$ minus the mean $m_{1}(t)$ of the envelope, $m_{1}(t)=\left(e_{\min }(t)+e_{\max }(t)\right) / 2$, and the first component $h_{1}(t)=X(t)-m_{1}(t)$ is obtained.

Step2. check whether $h_{1}(t)$ is IMF. If not, then return to step 1 , and take $h_{1}(t)$ as a new signal for screening. Repeat screening $k$ times, until $h_{1 k}(t)$ becomes IMF, take $c_{1}(t)=h_{1 k}(t)$. Residual signal is $r_{1}(t)=X(t)-c_{1}(t)$.

Step3. take $r_{1}(t)$ as a new signal, re perform step 1 to 2 , get the new IMF $c_{2}(t)$ and the residual volume $r_{2}(t)$. Repeat iteration n times: $r_{n}(t)=r_{n-1}(t)-c_{n}(t)$, until $r_{n}(t)$ is a monotonic function, EMD is over.

EMD is an adaptive filtering process, and the signal is decomposed adaptively according to the frequency scale, a series of narrow band signals are obtained from large to small frequency. Due to the completeness of EMD, the original signal $X(t)$ can be expressed as the sum of $n$ IMF and an average trend component $r_{n}(t)$.

\section{The normalized Hilbert Huang transform (NHHT)}

The normalized Hilbert- Huang transform (NHHT) is an improved Hilbert- Huang transform (HHT), it is composed of empirical mode decomposition (EMD) and normalized Hilbert transform (NHT).

For any continuous time signal $X(t)$, Hilbert transform is as follow:

$$
Y(t)=\frac{1}{\pi} \int_{-\infty}^{+\infty} \frac{X(\tau)}{t-\tau} \mathrm{d} \tau
$$

$X(t)$ and $Y(t)$ form complex conjugate pairs, and constitute an analytical signal as follows:

$$
\begin{gathered}
Z(t)=X(t)+j Y(t)=\sqrt{X^{2}(t)+Y^{2}(t)} e^{j \theta(t)} \\
\theta(t)=\arctan \frac{Y(t)}{X(t)}
\end{gathered}
$$

According to the derivative definition of the analytic signal phase, the instantaneous frequency is as follow: 


$$
f(t)=\frac{1}{2 \pi} \frac{\mathrm{d} \theta(t)}{\mathrm{d} t}
$$

It can be seen that $f(t)$ is a single valued function of $t$, the frequency changes with time, in order to make the instantaneous frequency meaningful, the time series of the Hilbert transform must be monotonous, and the IMF after the EMD just meets the requirement.

The definition of Hilbert spectrum (united timefrequency amplitude spectrum)[22] is:

$$
H(\omega, \mathrm{t})=\operatorname{Re} \sum_{i=1}^{k} z_{i}(\mathrm{t})=\operatorname{Re} \sum_{i=1}^{k} a_{i}(t) e^{j \theta_{i}(t)}
$$

Hilbert marginal spectrum[23] is:

$$
H(\omega)=\int_{0}^{T} H(\omega, \mathrm{t}) d t
$$

In the formula (15), $T$ is the signal length. Based on the $H(\omega, \mathrm{t})$ energy distribution in time frequency space, the energy (amplitude) of all moments for a certain frequency is added to form the total energy (amplitude) of the signal, that is, the Hilbert marginal spectrum $H(\omega)$. But the frequency is not always present at all time, or only at a certain moment, it may appear several times in different or equal amplitude, this physical meaning is consistent with the physical meaning of the Fourier spectrum[24], but in the Fourier spectrum, the same magnitude is required for any frequency, which is easy to destroy the true frequency of the signal and appear false frequency.

Because of the limitation of Bedrosian principle, the amplitude can cause serious disturbance to frequency modulation, which affects the instantaneous frequency. In order to eliminate this limitation, a normalized alg orith $\mathrm{m}$ is adopted to decompose IMF into a unique empirical envelope (AM) and a carrier (FM), and then Hilbert transform for carrier is done for Instantaneous frequency[25]. The essence is a kind of reverse frequency modulation and amplitude modulation. Specific steps are as follows:

Step1. The maximum value of the absolute value of each IMF component is solved, and the endpoint is also regarded as an extreme value.

Step2. The three spline is used to fit the maximum value curve $e_{1}(t)$. When the envelope is lower than the original data sequence, the local a rea envelope is replaced by a straight line envelope, use envelope normalized data $f_{1}=\operatorname{imf} / e_{1}, f_{1}$ is used as the new IMF.

Step3. Repeat steps 1, 2 until the absolute value of IMF is no more than 1 , and eventually will obtain its frequency modulation (carrier) component $f_{n}$ and amplitude modulation components $a(t)=e_{1} * e_{2} * \cdots * e_{n}$.

Step4. In order to obtain the instantaneous frequency, the Hilbert transform is carried out for the carrier.

United time-frequency amplitude spectrum of the signal is obtained based-on amplitude modulation component and instantaneous frequency, and then the Hilbert marginal spectrum is obtain.

\section{Analysis of an example}

As a time-frequency analysis method, of course, NHHT is most commonly used in nonlinear nonstationary signal analysis. Use NHHT to analyze nonlinear and non-stationary AM and FM signal in the formula (16):

$$
Y=\sin (30 \pi t)+10 t \cos (70 \pi t+\sin (20 \pi t))+\sin \left(30 \pi t^{2}\right)
$$

By the formula (16), it is known that the signal is composed of a $15 \mathrm{~Hz}$ sinusoidal signal, a linear FM signal and an AM \& FM signal. Through the FFT analysis, the Fourier spectrum (Fig.2) was obtained, and the amplitude peak values were appeared at $15 \mathrm{~Hz}, 25 \mathrm{~Hz}, 35 \mathrm{~Hz}, 45 \mathrm{~Hz}$, $55 \mathrm{~Hz}$ frequency in Fourier spectrum, and the amplitude of $35 \mathrm{~Hz}$ was the largest.

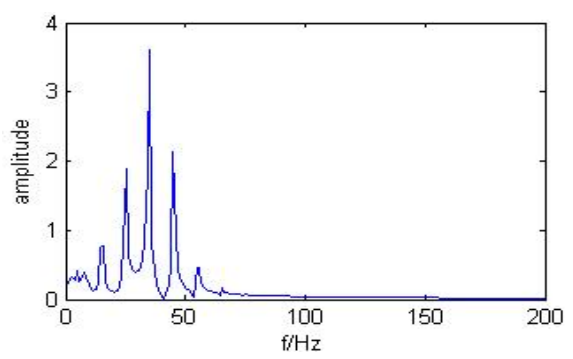

Fig.2. Fourier spectrum of the simulation signal

Use NHHT to analy ze the signal, firstly EMD (Fig. 3) is done, the original signal is decomposed into a series of IMF and residual RES, IMF is a series of signal components with different frequency components. According to the approximate orthogonality of EMD, the IMF is nearly orthogonal to each other, that is, the frequency components of each IMF component are almost different.

In Fig.3, each IMF is arranged according to its frequency components fro $m$ the big to small arrangement, and due to the residual RES is very small, the decomposition is more successful. Seen from Fig.3, the signal is made up of three parts: the $15 \mathrm{~Hz}$ constant frequency component, linear frequency modulation component, as well as frequency oscillation component by taking $35 \mathrm{~Hz}$ as the center and $10 \mathrm{~Hz}$ as the oscillation amplitude.

NHT is done for the IMF obtained by EMD, and united time-frequency amp litude spectrum (Fig.4) is obtained. In the united time-frequency amplitude spectrum, the amplitude changes of the same frequency can be seen at different times, this is not done in the Fourier analysis which is required to be based on the same frequency and its amplitude can not be changed with time.

Seen from Hilbert marginal spectrum (Fig.5), there is no more than $50 \mathrm{~Hz}$ frequency band, and the frequency components of $55 \mathrm{~Hz}$ appear in the Fourier spectrum. According to the theoretical analysis, in a second the signal frequency can not be greater than $50 \mathrm{~Hz}$, the false 
frequency appears in the Fourier spectrum, actual results show that the FFT is not suitable for analy zing nonlinear signals and appear spectrum leakage, and NHHT is not restricted, these show, compared to FFT and other conventional algorithms, NHHT in analyzing nonlinear signal has incomparable superiority.

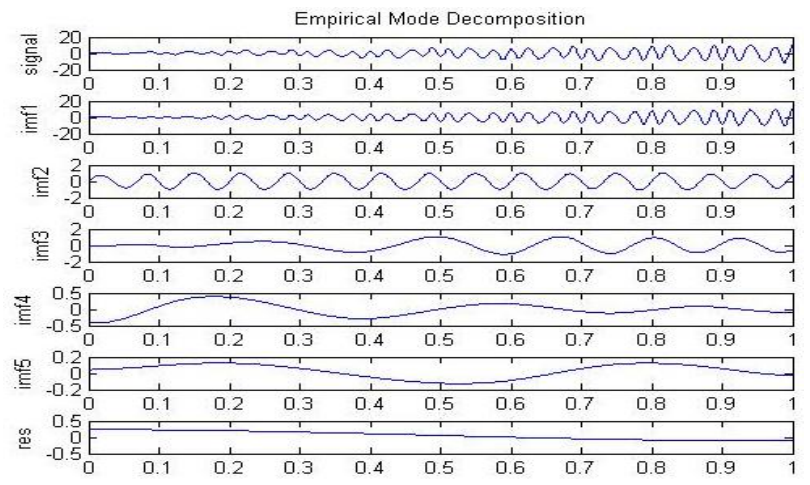

Fig.3. EMD of the simulation signal

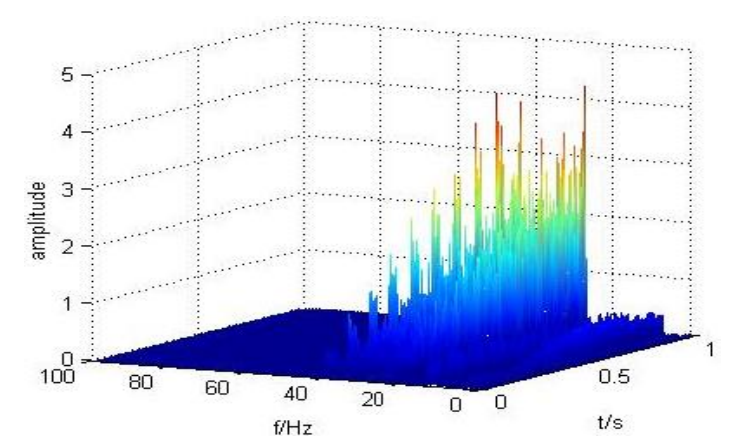

Fig.4. United time-frequency amplitude spectrum of the simulation signal

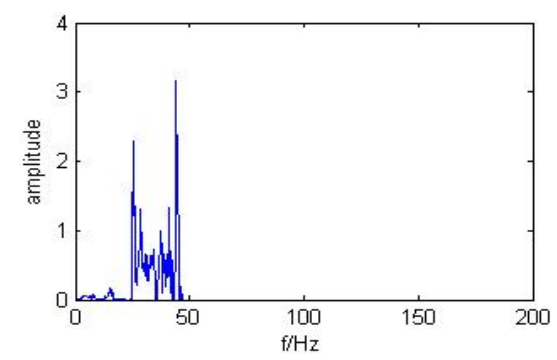

Fig.5. Hilbert marginal spectrum of the simulation signal

\section{Simulation AND ANAlysis OF Single PhaSE GROUNDING FAULT}

\section{A. Construction of single phase grounding fault system}

EMTP is the first of the electromagnetic transient analysis software produced by Tan Weier, Canada, which is a powerful tool for power system simulation, which can be used to analyze the steady and transient characteristics of power system. The typical application of EMTP is to simulate the change of electrical parameters with time under the disturbance in power system, such as the change of the feeder current after the occurrence of grounding fault in the power grid.
ATP is the program package programmed by the Danish scholar Hart based-on the use of EMTP for the kernel. Because ATP uses the Windows interface, it has a more friendly man-machine window compared with the traditional EMTP, the application is more simple. In ATP, the simulation of 5 output lines is built, and the topological structure of the model is shown in Fig.6.

Fig. 6 simulation model is a $10 \mathrm{kV}$ three-phase symmetric system model, the line uses the distribution parameters module with 5 outlets. In Fig.6, $10 \mathrm{kV}$ voltage is obtained by $110 \mathrm{kV}$ AC 3 phase power supply through the $110 / 10 \mathrm{kV}$ transformer, the transformer is Yy 0 . It is not the grounding system when the $\mathrm{K}$ is open, and is the grounding system through the arc suppression coil when the $\mathrm{K}$ is closed.

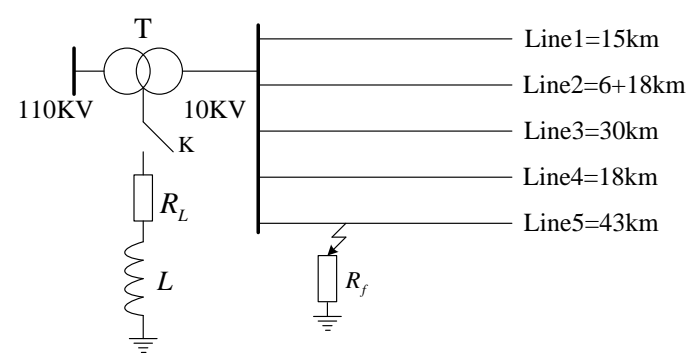

Fig.6. Simulation model of small current grounding fault

In Fig.6, the parameters of the small current fault grounding system are shown as follows: Line L1 is $15 \mathrm{~km}$ pure cable line. Line L2 is a mixed line of overhead line and cable, the length of which is $18 \mathrm{~km}$ and $6 \mathrm{~km}$ respectively. L3, L4 and L5 are the pure overhead lines, the length of which is $30 \mathrm{~km}, 18 \mathrm{~km}$ and $43 \mathrm{~km}$.

Cable line parameters are:

Positive sequence impedance $Z_{1}=(0.125+j 0.095) \Omega / \mathrm{km}$, Zero sequence impedance $Z_{0}=(0.97+j 1.590) \Omega / \mathrm{km}$.

Positive sequence susceptance $b_{1}=83.566 \mu \mathrm{s} / \mathrm{km}$, Zero sequence susceptance $b_{0}=83.566 \mu \mathrm{s} / \mathrm{km}$.

The parameters of the overhead line are:

Positive sequence impedance $Z_{1}=(0.270+j 0.351) \Omega / \mathrm{km}$, Zero sequence impedance $Z_{0}=(0.475+j 1.757) \Omega / \mathrm{km}$.

Positive sequence susceptance $b_{1}=3.267 \mu \mathrm{s} / \mathrm{km}$, Zero sequence susceptance $b_{0}=1.100 \mu \mathrm{s} / \mathrm{km}$.

The terminal load of each outlet is $1000+j 20 \Omega$.

The capacitance current of single phase to ground can be calculated as follow:

$$
I_{c}=3 \omega C_{0 \Sigma} U_{\varphi}=32.46(\mathrm{~A})>20(\mathrm{~A})
$$

In general, when the capacitance current is more than $20 \mathrm{~A}$ in the $10 \mathrm{KV}$ medium voltage power grid, it is necessary to install the arc suppression coil. The inductance of the arc suppression coil is obtained by using formula (18):

$$
\eta \% \omega L=\frac{1}{3 \omega C_{0 \Sigma}}
$$


The arc suppression coil is set by the over compensation of $\eta \%=110 \%$, through calculation, the inductance of arc suppression coil is $L=0.55 \mathrm{H}$. The value of series resistance is set to $R_{L}=17.28 \Omega$ by the $10 \%$ arc suppression coil inductance.

\section{B. Time frequency analysis of zero sequence transient current based on $H H T$}

\section{1) Extraction of transient signal}

From the previous analysis, the transient grounding current includes the steady state power frequency component and transient component of grounding current. According to the superposition principle, the power frequency component in the fault transient zero sequence current of each feeder is the superimposition of the asymmetric component before the fault and the steady state power frequency component after the fault. And because the actual small current grounding fault occurs after the 5 6 cycle, as shown in Fig.7 (a), the zero sequence current transient component has been quite small, it is considered that the electromagnetic transient process is basically the end. Therefore, a reasonable time window is designed to filter the steady-state component, its window size is a power frequency cycle, the expression of the zero sequence current transient component is as follow:

$$
i_{0 k f}(t)=i_{0 k}(t)-i_{0 k}(t+7 T)
$$

In the formula (19), $i_{0 k}(t)$ is the zero sequence current of the $k$ feeder, and $T$ is the power frequency cycle.

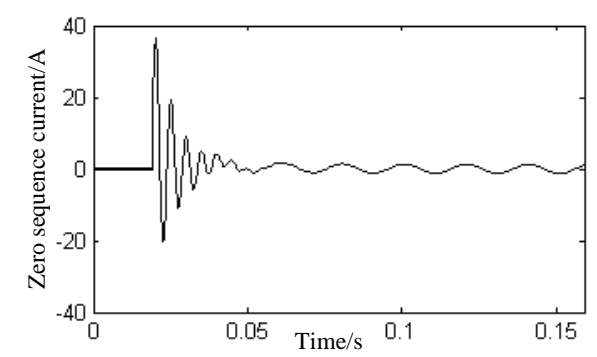

(a) The zero sequence current of fault line

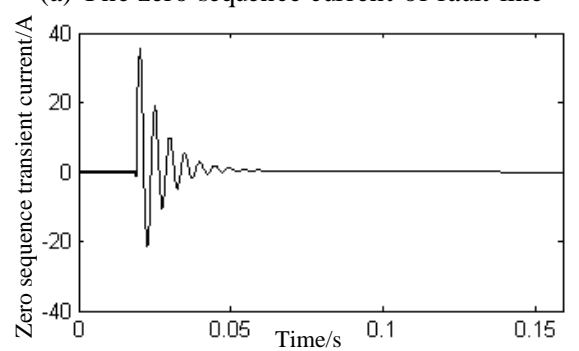

(b) The Zero sequence transient current of fault line

Fig.7. The extraction of fault signal transient component

In Fig.7 (a), the grounding fault occurs in the feeder L5 at the bus terminal $12 \mathrm{~km}$ and the fault phase $60^{\circ}$, the ground resistance is $2 \Omega$. The actual zero sequence current flows through the feeder L5, which contains the steady state and transient components. Can be seen after the above treatment, it is very good to filter out the steady-state component of the fault current, the transient component is obtained as shown in Fig.7 (B).

\section{2) Transient time frequency analysis of zero sequence} transient current

HHT is done for the zero sequence transient current signal in Fig.7 (b). First, EMD is done for the zero sequence transient current, seen from Fig.8 (a), the current signal which contains a variety of frequency is decomposed into a series of different center frequency current components, namely IMF1 to IMF6, its time scale is getting bigger and bigger, that is, the center frequency of each IMF frequency band is gradually reduced. The center frequency and bandwidth of each current component are adaptive, without a priori decomposition basis function, the frequency components of each IMF component are not equal in theory, and the amplitude of each IMF component is determined by its time and frequency. From the ratio of the EMD residual amplitude and the original signal, the decomposition is more successful. From Fig.8 (a), the main frequency components of the signal are concentrated in IMF4, the amplitude of the other IMF components are very s mall which compared to IMF4, also the frequency is about $190 \mathrm{~Hz}$ by Hilbert transform for IM4, which is relative to the Hilbert marginal spectrum of Fig.8 (c).

The united time-frequency amplitude spectrum is obtained by the normalized Hilbert transform (NHT) for the IMF component of the decomposition, that is, the Hilbert spectrum (Fig.8 (b)). From Hilbert spectrum, it can be seen that the distribution of the frequency components of the transient current in the whole time frequency space, the main frequency band of the transient current is concentrated in the low frequency band between 0 and $500 \mathrm{~Hz}$. The high frequency components of the frequency components are relatively scattered and mainly concentrated in the moment of failu re occurrence. In the Hilbert spectrum, the amplitude is s maller but the power frequency steady-state periodic component is found in the whole analysis. This corresponds to IMF6 after EMD. From Fig.8(a), it can be seen the frequency of IMF6 is $50 \mathrm{~Hz}$, and the analysis of the transient current in the last part shows that this is the function of the system inductance. The Hilbert spectrum shows that the signal is attenuated in the whole, and the performance of the first $1 / 4$ power frequency cycle is obvious, which is consistent with the transient component of zero sequence current.

The amplitude spectral of Hilbert is integrated in the whole analysis, and the amplitude of each frequency component at each moment is accumulated, the Hilbert marginal spectrum is obtained. This is a kind of spectrum of signal energy distribution, it is a kind of characterization form of frequency and energy, and the amplitude will be normalized in the practical application. Seen from Fig. 8 (c) of the Hilbert marg inal spectrum, its frequency components are relatively concentrated around $200 \mathrm{~Hz}$, the main energy distribution of the signal is between $0-500 \mathrm{~Hz}$. 


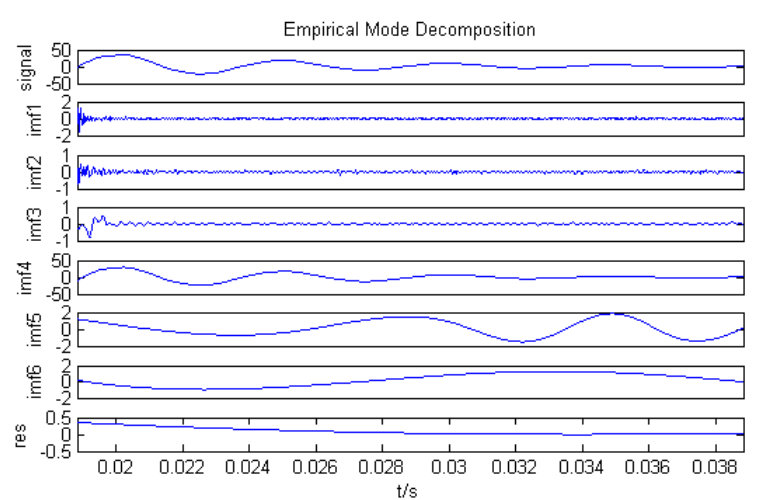

(a) Empirical mode decomposition of zero sequence transient current

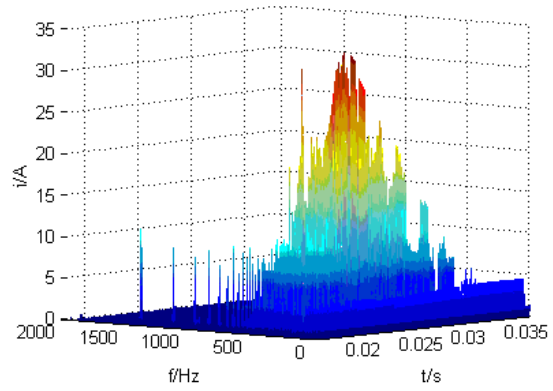

(b) United time-frequency amplitude spectrum of zero sequence transient current

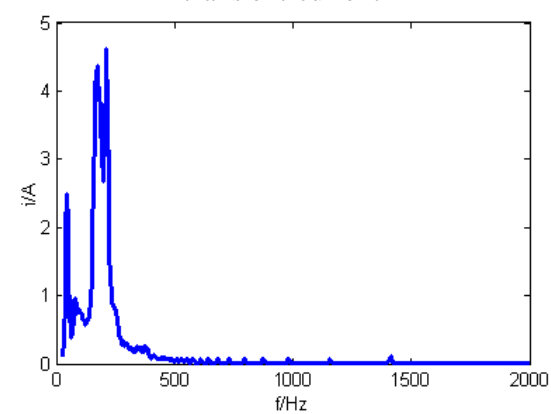

(c) Hilbert marginal spectrum of zero sequence transient current

Fig.8. Transient time frequency analysis of fault line(L5) zero sequence current

\section{The influence of main factors on the transient time- frequency characteristics}

The main factors that affect the fault signal are the structure of the circuit, the fault phase, the grounding resistance, the distance between the fault point and the bus, among them, the fault phase and grounding resistance are the dominant factors. In this paper, the effects of the fault phase and the grounding resistance on the time-frequency characteristics of the signal are discussed.

\section{1) The influence of the fault phase on the transient time- frequency characteristics}

Under the condition of grounding resistance 5, by changing the fault phase, the zero sequence current of a series of fault feeder (L5) is obtained. The transient component is obtained by the previously designed filter window, the time-frequency characteristics of the current signal are obtained by HHT analysis, as shown in Fig.9.

Fig. 9 is time frequency characteristics of the transient component of zero sequence current when the fault phase is changed at fault line (L5). From the graph, we can know that with the increase of the fault phase, the DC component of the current decreases rapidly, and the amplitude of the current is obviously increased, which is consistent with the conclusion of the theoretical analys is. It can be seen from the Hilbert spectrum of different fault phase, the amplitude of the main vibration mode of the current signal is exponential decay with the increase of time. With the increase of the phase, the frequency components of the current are becoming more and more abundant, and the marginal spectrum of Hilbert describes this phenomenon in the frequency domain.

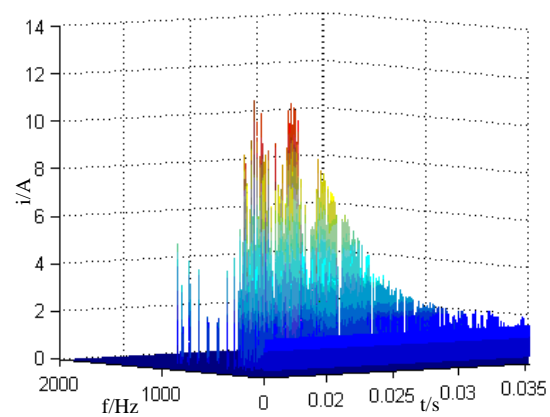

(a) Hilbert amplitude spectrum at 00

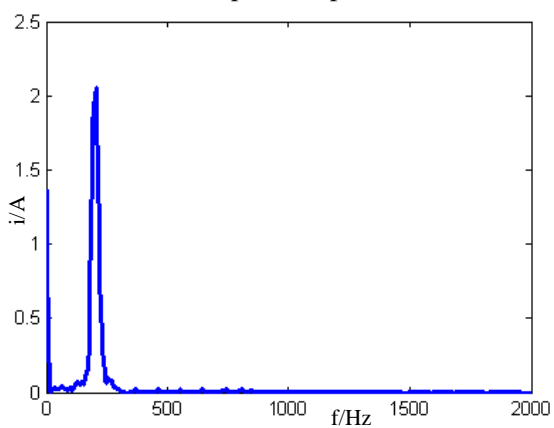

(b) Hilbert marginal spectrum at 00

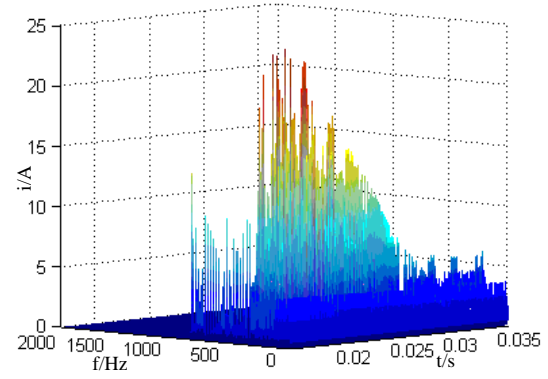

(c) Hilbert amplitude spectrum at 300

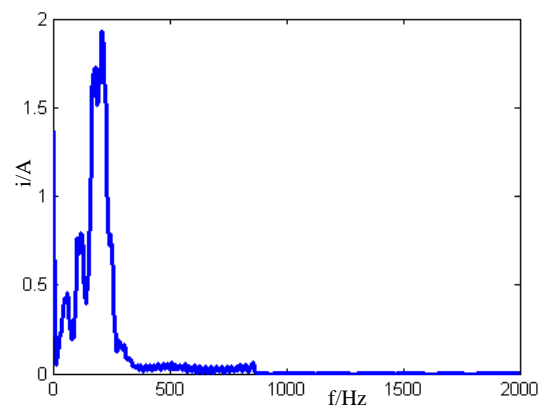

(d) Hilbert marginal spectrum at 300 


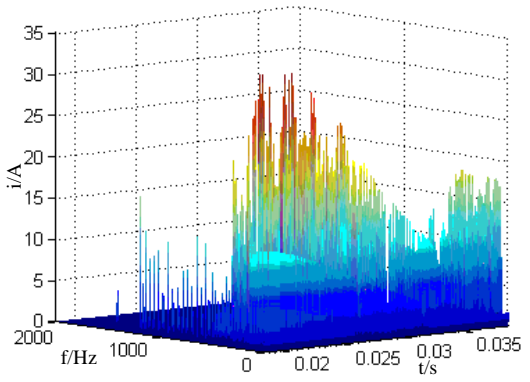

(e) Hilbert amplitude spectrum at 600

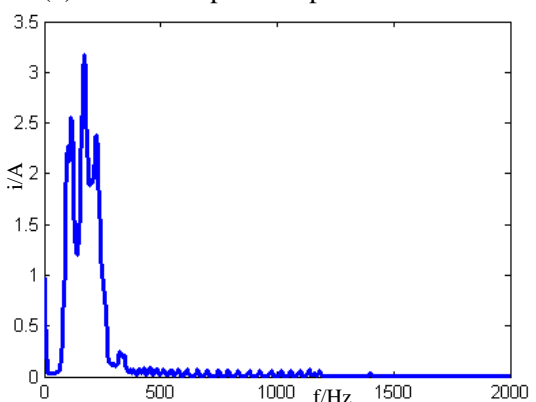

(f) Hilbert marginal spectrum at 600

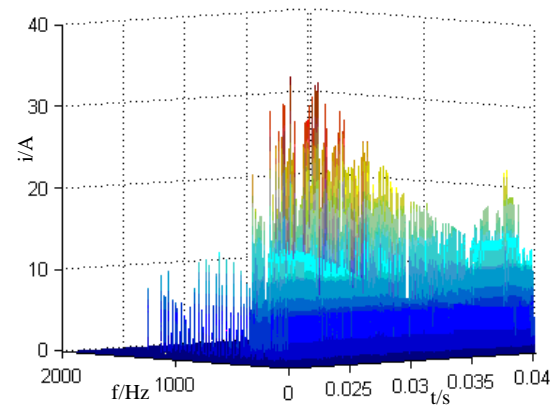

(g) Hilbert amplitude spectrum at 900

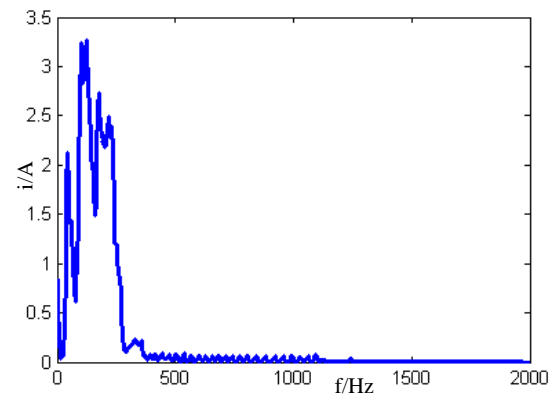

(h) Hilbert marginal spectrum at 900

Fig.9. The influence of the fault phase on the transient time-frequency characteristics of fault line zero sequence current

In short, with the increase of the phase, the amplitude and frequency components of the signal increase, the distribution of the signal energy will be more and more dispersed.

2) The influence of the grounding resistance on the transient time-frequency characteristics

In [26], the effects of very-high resistance grounding on the selectivity of ground-fault relaying are studied.

The fault resistance and fault phase are the main factors that affect the zero sequence current. Set the fault phase at $90^{\circ}$, change the grounding resistance, analyze the signal time-frequency characteristics of different grounding resistance.
Fig.10 is Hilbert spectrum and Hilbert marginal spectrum of the fault line at $50 \Omega, 100 \Omega, 500 \Omega, 5000 \Omega$. It can be seen from the figure that with the increase of grounding resistance, signal current amplitude and frequency components gradually reduced. At the $50 \Omega$ around, the system has been in a state of damping, and the frequency components of the system are relatively concentrated in Fig.10 (b). And with the increase of the grounding resistance, the system is re entered into an under damping condition, as shown in Fig.10 (d), although the main frequency components of the system are concentrated in $50 \mathrm{~Hz}$, but the energy of $150 \mathrm{~Hz}$ and $250 \mathrm{~Hz}$ is also larger, and the system energy is relatively dispersed. After a further increase in the grounding resistance, the current signal frequency is further concentrated in the low frequency part, as shown in Fig.10 (f), and the signal energy becomes more concentrated. When the grounding resistance reaches $5000 \Omega$ values, it almost becomes a DC component, as shown in Fig.10 (h).

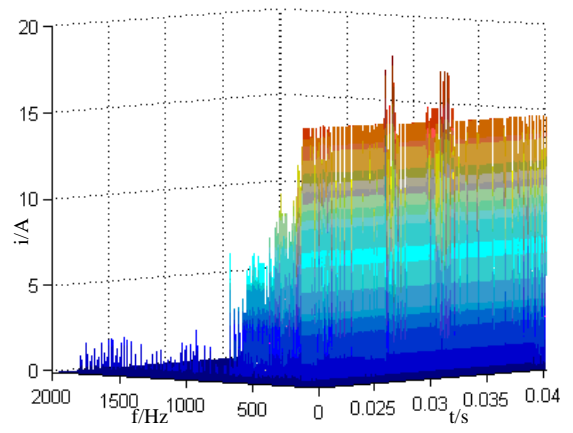

(a) Hilbert amplitude spectrum at $50 \Omega$

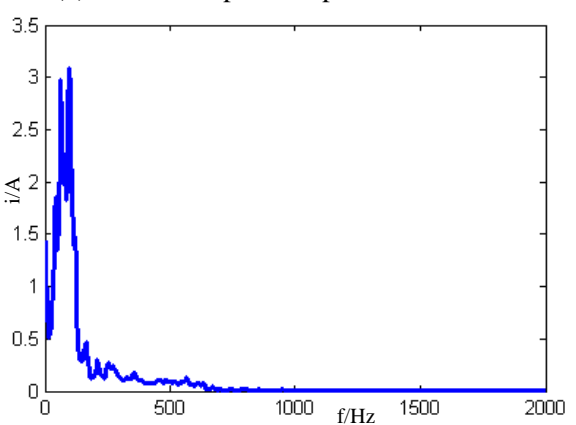

(b) Hilbert marginal spectrum at $50 \Omega$

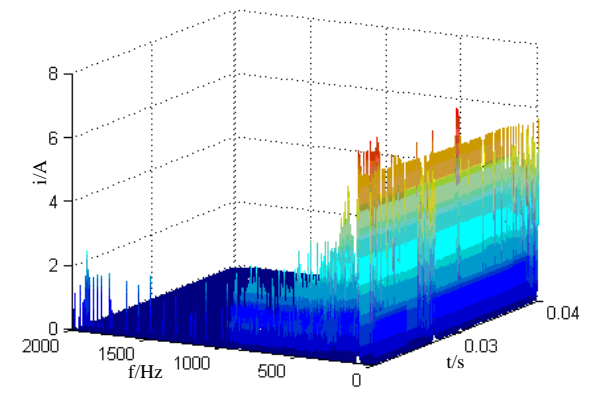

(c) Hilbert amplitude spectrum at $100 \Omega$ 


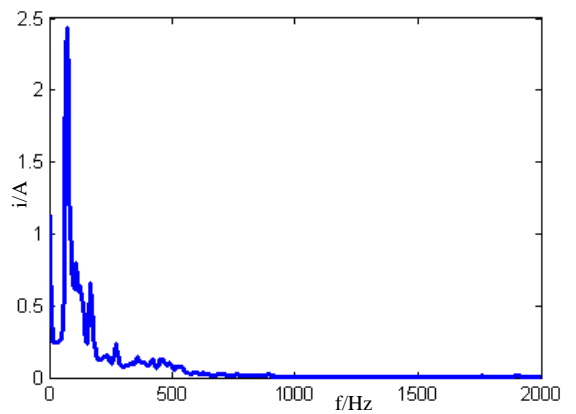

(d) Hilbert marginal spectrum at $100 \Omega$

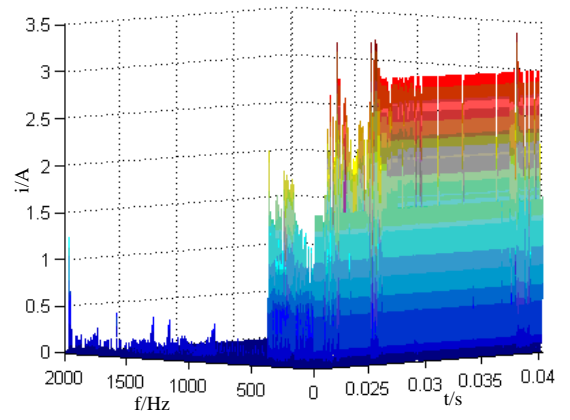

(e) Hilbert amplitude spectrum at $500 \Omega$

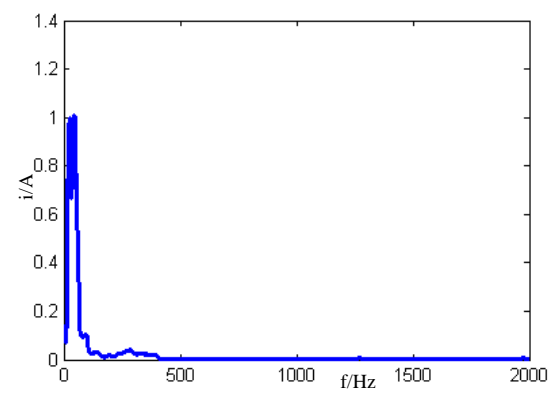

(f) Hilbert marginal spectrum at $500 \Omega$

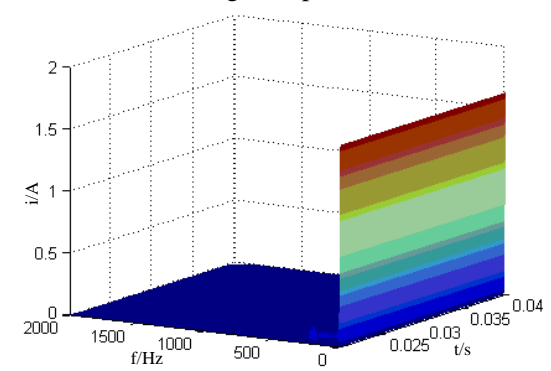

(g) Hilbert amplitude spectrum at $5000 \Omega$

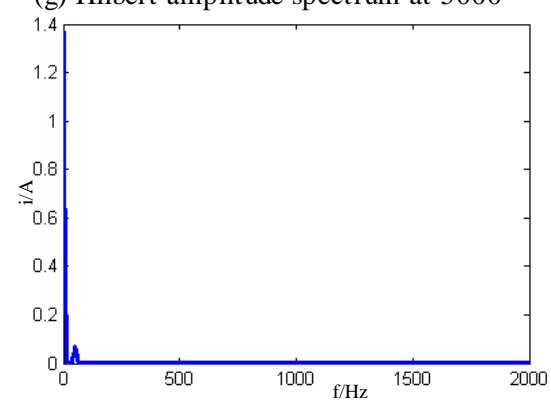

(h) Hilbert marginal spectrum at $5000 \Omega$

Fig. 10. The influence of the grounding resistance on the transient timefrequency characteristics of fault line zero sequence current

In short, with the continuous increase of the grounding resistance, the system undergoes a sate of less damping, over damping, and then to less damping, the signal is towards the low frequency in general, the frequency components are more and more concentrated, and the energy distribution is becoming more and more concentrated.

\section{CONCLUSION}

In this paper, the transient analysis of single-phase grounding current in small current grounding power grid is carried out. Resultly the grounding current is mainly capacitive current and inductance current. On this basis, the theoretical analysis and simulation are done. According to the different fault conditions, such as feeder structure, fault phase, grounding resistance, fault distance, etc, the simulation results show that the fault phase and the grounding resistance have most influence on the feeder zero sequence current.

The main results of this paper are as follows:

(1) Analyze the steady-state and transient characteristics of single phase grounding fault in the small current grounding power grid, in which the neutral point is not connected to ground or connected to ground through the arc suppression coil.

(2) Under the EMTP_ATP environment, a small current grounding system is built, and the simulation analysis of the single-phase grounding fault under different conditions is done. According to the power system related theory calculation result, the system grounding capacitance over $10 \mathrm{KV}$ voltage level is allowed the maximum capacitance current 20A, the neutral point must be connected with the arc suppression coil, and the compensation degree is $10 \%$.

(3) HHT is applied to the single phase grounding fault detection through the arc suppression coil. Firstly, a time window is selected to extract the transient component of the fault signal, and the transient component of the feeder zero sequence current is extracted, and the time frequency analysis of transient signal is done with the HHT. The two main factors that influence the transient signal, the fault phase and the grounding resistance, are studied respectively.

\section{ACKNOWLEDGMENT}

The authors wish to thank my graduate tutor Hongbin Wu Professor and Benxian Xiao Professor and graduate student Lin Zhang.

\section{REFERENCES}

[1] N.E.Huang, Z.Shen, S.R.Long, et al., "The empirical mode decomposition and the Hilbert spectrum for nonlinear and non-stationary time series analy sis," Proc $R$ Soc Lond A, 454, pp.903-995, 1998.

[2] Vasudevan K, Cook FA., "Empirical mode skeletonization of deep crustal seismic data: Theory and applications," Journal of Geophysical Research-Solid Earth, Vol.105(B4), APR 10, pp.7845-7856, 2000.

[3] Loh CH, Wu TC, Huang NE., "Application of the empirical mode decomposition-Hilbert spectrum method to identify near-fault ground-motion characteristics and 
structural responses," Bulletin of the Seismological Society of America, Vol.91, No.5, pp.1339-1357, 2001.

[4] Xun Zhu, Zheng Shen, Stephen D.E, et al., "Gravity wave characteristics in the middle atmosphere derived from the empirical mode decomposition method," Journal of Geophysical Research, Vol.102, No.D14, pp.16545$16561,1997$.

[5] Yang Jann N, L.Y., "System identification of linear structures using Hilbert transform and Empirical Mode Decomposition," Proceedings of the International Modal Analysis Conference, pp.213-219, 2000.

[6] Komm RW, Hill F. Howe R., "Empirical mode decomposition and Hilbert analysis applied to rotation residuals of the solar convection zone," Astrophysical Journal, Vol.558, No.1, pp.428-441, 2001.

[7] Ranjit A. Thuraisingham, "Estimation of Teager energy using the Hilbert-Huang transform," IET Signal Process, Vol.9, No.1, pp.82-87, 2015.

[8] Dhouha Kbaier Ben Ismail, Pascal Lazure, In grid Puillat, "Advanced Spectral Analysis and Cross Correlation Based on the Empirical Mode Decomposition: Application to the Environmental Time Series," IEEE Geoscience and Remote Sensing Letters, Vol.12, No.9, pp.1968-1972, 2015.

[9] Liang H, Lin Z, McCallum RW., "Artifact reduction in electrogastrogram based on empirical mode decomposition method," Medical\& Biological Engineering \& Computing, Vol.38, No.1, pp.35-41, 2000.

[10] Echeverria J C,Crowe J A,Woolfson M S,Hayes-Gill B R., "Application of empirical mode decomposition to heart rate variability analysis," Medical\& Biological Engineering \& Computing, 39(2): 471-479, 2001.

[11] Abdenour Soualhi, Kamal Medjaher, Noureddine Zerhouni, "Bearing Health Monitoring Based on HilbertHuang Transform, Support Vector Machine, and Regression," IEEE Transactions on Instrumentation and Measurement, Vol.64, No.1, pp.52-62, 2015.

[12] Helong Li, Sam Kwong, Lihua Yang, Daren Huang, Dongping Xiao, "Hilbert-Huang Transform for Analysis of Heart Rate Variability in Cardiac Health," IEEE/ACM Transactions on Computational Biology and Bioinformatics, Vol.8, No.6, pp.1557-1567, 2011.

[13] Amin Yazdanpanah Goharrizi, Nariman Sepehri, "Internal Leakage Detection in Hydraulic Actuators Using Empirical Mode Decomposition and Hilbert Spectrum," IEEE Transactions on Instrumentation and Measurement, Vol.61, No.2, pp.368-378, 2012.

[14] Chao Wang, Xiao Liu, Zhe Chen, "Incipient Stator Insulation Fault Detection of Permanent Magnet Synchronous Wind Generators Based on Hilbert-Huang Transformation," IEEE Transactions on Magnetics, Vol.50, No.11, pp.1-4, 2014.

[15] Antonio Garcia Espinosa, Javier A. Rosero, Jordi Cusid’o, Luis Romeral, Juan Antonio Ortega, "Fault Detection by Means of Hilbert-Huang Transform of the Stator Current in a PMSM With Demagnetization," IEEE Transactions on Energy Conversion, Vol.25, No.2, pp.312-318, 2010.

[16] Shu Hongchun, Tian Xincui, Dai Yuetao, "The Identification of Internal and External Faults for $\pm 800 \mathrm{kV}$ UHVDC Transmission Line Using Wavelet based MultiResolution Analysis," I.J. Intelligent Systems and Applications, 3(03): 47-53, 2011.

[17] Ashwani Kumar Narula, Amar Partap Singh, "Fault Diagnosis of Mixed-Signal Analog Circuit using Artificial Neural Networks," I.J. Intelligent Systems and Applications, 7(07): 11-17, 2015.

[18] A. R. Messina, Vijay Vittal, "Nonlinear, Non-Stationary
Analysis of Interarea Oscillations via Hilbert Spectral Analy sis," IEEE Transactions on Power Systems, Vol.21, No.3, pp.1234-1241, 2006.

[19] Mohammad Jasa Afroni, Danny Sutanto, David Stirling, "Analysis of Nonstationary Power-Quality Waveforms Using Iterative Hilbert Huang Transform and SAX Algorithm," IEEE Transactions on Power Delivery, Vol.28, No.4, pp.2134-2144, 2013.

[20] Patrick Flandrin, "Empirical Mode Decomposition as a Filter Bank," IEEE Signal Processing Letters, Vol.11, No.2, pp.431-435, 2004.

[21] Gabriel Rilling, "On the Influence of Sampling on the Empirical Mode Decomposition," IEEE Signal Processing Letters, Vol.17, No.4, pp.214-218, 2005.

[22] Xiaonan Hui, Shilie Zheng, Jinhai Zhou, Hao Chi, Xiaofeng Jin, Xianmin Zhang, "Hilbert-Huang Transform Time-Frequency Analysis in $\varphi$-OTDR Distributed Sensor," IEEE Photonics Technology Letters, Vol.26, No.23, pp.2403-2406, 2014.

[23] Yingjun Yuan, Zhitao Huang, Hao Wu, Xiang Wang, "Specific emitter identification based on Hilbert-Huang transform-based time-frequency-energy distribution features," IET Commun., Vol.8, No.13, pp.2404-2412, 2014.

[24] Zhaohua Wu, Norden E.Huang, "Ensemble Empirical Mode Decomposition: a noise assisted data analysis method," Advances in Adaptive Data Analysis, 1(1):1-41, 2009.

[25] Norden E. Huang, Zhaohua Wu, "On Instantaneous Frequency," Advances in Adaptive Data Analysis, Vol.1, No.2, pp.177-229, 2009.

[26] Thomas N., "The effects of very-high resistance grounding on the selectivity of ground-fault relaying in high-voltage long wall power systems," IEEE Trans Ind Appl., Vol.37, No.2,pp.398-406, 2001.

\section{Authors' Profiles}

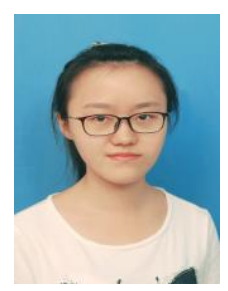

Yingwei Xiao was born in Hefei, China in 1993. She has completed the B.Sc degree in electrical engineering and automation from Anhui Polytechnic University, Wuhu, China in 2015. Currently she is still pursuing her master degree in Hefei University of Technology, Hefei, China. Her research interests include automation of electric power system, micro grid power flow calculation.

How to cite this paper: Yingwei Xiao, "Characteristic Research of Single-Phase Grounding Fault in Small Current Grounding System based-on NHHT", International Journal of Intelligent Systems and Applications (IJISA), Vol.8, No.12, pp.46-56, 2016. DOI: 10.5815/ijisa.2016.12.06 